PUIATTI, M.; KATSUMOTO, R.; PEREIRA, F.H.F.; BARRELLA, T.P. Crescimento de plantas e produção de rizomas de taro 'Chinês' em função do tipo de muda. Horticultura Brasileira, Brasília, v. 21, n. 1, p. 110-115, março 2003.

\title{
Crescimento de plantas e produção de rizomas de taro 'Chinês' em fun- ção do tipo de muda
}

\author{
Mário Puiatti ${ }^{1}$; Rumi Katsumoto; Francisco H. F. Pereira ${ }^{1}$; Tatiana P. Barrella ${ }^{1}$ \\ ${ }^{1 /}$ UFV - Depto. de Fitotecnia 36.571-000 Viçosa-MG; E-mail: mpuiatti@ufv.br
}

\begin{abstract}
RESUMO
Verificou-se a viabilidade agronômica e econômica da utilização do rizoma mãe (cabeça) de taro (Colocasia esculenta) 'Chinês' na implantação da cultura, comparado à propagação convencional (rizomas filhos). O experimento constou de dez tratamentos, dispostos no delineamento blocos ao acaso, com cinco repetições. Os tratamentos consistiram de cabeça intacta, seis segmentos da cabeça e de três classes de rizomas filhos intactos, a saber: $\mathrm{CI}=$ cabeça intacta (peso médio de $300 \mathrm{~g}$ ); CMS = metade superior da cabeça $(150 \mathrm{~g}) ; \mathrm{CMI}=$ metade inferior da cabeça $(150 \mathrm{~g}) ; \mathrm{CTS}=$ terço superior da cabeça $(100 \mathrm{~g}) ; \mathrm{CTM}=$ terço mediano da cabeça $(100$ g); CTI = terço inferior da cabeça $(100 \mathrm{~g}) ; \mathrm{CML}=$ metade lateral da cabeça $(150 \mathrm{~g}) ; \mathrm{RFG}=$ rizoma filho grande $(100 \mathrm{~g}) ; \mathrm{RFM}=$ rizoma filho médio $(50 \mathrm{~g})$ e RFP = rizoma filho pequeno $(25 \mathrm{~g})$. Durante o ciclo avaliou-se, mensalmente, o estande, a altura e área foliar das plantas; na colheita, avaliou-se a produção de rizomas mãe e das classes de rizomas filhos. Realizou-se a análise econômica estimando-se os possíveis retornos obtidos com a venda de cada classe de rizomas. As menores porcentagens de plantas emergidas foram observadas nos tipos de mudas CTI, CMI e CTM com, respectivamente, $0 \%, 35 \%$ e $68 \%$. Aos 105 e 165 dias após plantio foram observados os valores máximos em índice de área foliar e altura de plantas, respectivamente. A utilização da cabeça intacta (CI) e dos segmentos CMS, CML e CTS apresentaram-se viáveis agronômica e economicamente, com rendimentos e retorno financeiro das classes de rizomas mãe, filho médio, comercial, refugo e total, semelhantes aos proporcionados pelos tipos de mudas RFG e RFM. Segmentos CML e CTS e muda RFG proporcionaram, como grande vantagem, maiores participações da classe filho grande nos rendimentos de rizomas por área e retorno estimado, que os demais tipos de mudas. A utilização da cabeça intacta e/ou dos segmentos CMS, CML e CTS, na propagação do taro 'Chinês', é vantajosa, sobretudo em situação de não haver mercado para o comércio de cabeça.
\end{abstract}

Palavras-chave: Colocasia esculenta, propagação vegetativa, inhame, rendimento.

\begin{abstract}
Plant growth and production of 'Chinês' corms taro according to the propagation method
\end{abstract}

The agronomical and economic viability of the use of the 'Chinês' taro main corm (Colocasia esculenta) for crop implantation was checked, compared to the conventional cormels propagation. The experiment consisted of ten treatments, in the randomized complete block design, with five repetitions. The evaluated treatments were: use of the main corm (intact head or corm), by six head segments, and by three classes of intact cormels like: $\mathrm{IH}=$ Intact head (medium weigh, $300 \mathrm{~g}) ; \mathrm{SHH}=$ Superior half head $(150 \mathrm{~g}) ; \mathrm{IHH}=$ Inferior half head $(150 \mathrm{~g})$; STH = Superior third head $(100 \mathrm{~g})$; MTH = Medium third head $(100 \mathrm{~g})$; ITH = Inferior third head (100 g); SiHH $=$ Side half head $(150 \mathrm{~g}) ; \mathrm{BC}=$ Big cormels $(100 \mathrm{~g})$; $\mathrm{MC}=$ Medium cormels $(50 \mathrm{~g})$, and $\mathrm{SC}=$ Small cormels $(25 \mathrm{~g})$. At monthly intervals, the stand, height, and the leaf area of the plants were assessed. Corm yield and the classes of cormels were evaluated at harvest. The economic analysis was done estimating the possible returns obtained with the propagation method. The smaller $\%$ of emergency was observed in the seedling kinds: ITH, IHH and MTH, with $0 \%, 35 \%$ and $68 \%$, respectively. Maximum values of leaf area index and plant height were observed at 105 and 165 days after seedling. The use of intact head (IH) and segments SHH, SiHH and STH was agronomically and economically viable resulting in profitable corm yield and cormels classes, medium, commercial, reject and total, similar to the obtained by the kind of seedlings BC and MC. Segments $\mathrm{SiHH}, \mathrm{STH}$, and BC were advantageous because of higher percentage of bigger cormels class $\mathrm{BC}$ per unit area and higher economic return. Application of the intact head and of segments $\mathrm{SHH}, \mathrm{SiHH}$, and $\mathrm{STH}$, in taro propagation is of interest especially when there is no market demand for the head trade.

Keywords: Colocasia esculenta, vegetative propagation, dasheen, eddo, yield.

\section{(Recebido para publicação em 14 de março de 2002 e aceito em 10 de outubro de 2002)}

$\mathrm{O}$ iginário da Ásia, o taro [Colocasia esculenta (L.) Schott], também conhecido como inhame no centro-Sul do Brasil, é uma planta monocotiledônea da família Araceae (Plucknett, 1983). A planta adulta é constituída de um rizoma central (rizoma mãe ou cabeça central), do qual são formados, lateralmente, vários rebentos (rizomas filhos), órgãos, esses, de importância na exploração comercial. Dos rizomas, principalmente do rizoma mãe, saem longos pecíolos car- nudos, de comprimento e coloração variável com o clone, terminados por grandes limbos foliares em formato de coração (Puiatti, 2001).

O taro é considerado importante "hortaliça de raiz", com produção mundial estimada de 8,87 milhões de t (FAO, 2001), sendo a brasileira cerca de 225 mil t, em 1998 (Camargo Filho et al., 2001) e a mineira de 19,8 mil t em 2001 (Puiatti, 2001). Constitui-se em alimento amiláceo básico em diversas nações, principalmente da Ásia, África e ilhas do Pacífico (Wang, 1983). As características de adaptabilidade edafoclimáticas e rusticidade fazem do taro uma cultura típica de subsistência.

O componente principal dos rizomas é o amido, conhecido por apresentar grânulos relativamente pequenos, quando comparado a outras amiláceas (Nip, 1990), com digestibilidade da ordem de $97 \%$, proporcionando eficiente liberação dos componentes durante a diges- 
tão (Standal, 1983), fato que torna o taro indicado na alimentação de crianças, idosos e de convalecentes. Apesar dessas características, os rizomas centrais (cabeças) têm baixo valor de comercialização e normalmente são eliminados na propriedade rural devido ao sabor às vezes "aguado" e o aspecto visual pouco atraente aos olhos do consumidor (Puiatti, 2001).

A área foliar das plantas tem papel crucial na interceptação da radiação solar e acúmulo de biomassa. O desenvolvimento vegetativo da parte aérea do taro, lento no início, atinge o ponto máximo entre quatro a seis meses de ciclo. Essa fase é marcada pelo aumento da área foliar, do número e peso de folhas e altura da planta. Na fase seguinte, inicia-se o processo inverso, no qual as novas folhas serão cada vez menores com pequena taxa de crescimento, podendo este ser paralisado ao entrar em processo de maturação e/ou sob condições de temperatura abaixo do limiar (Heredia Zárate, 1988; Puiatti et al., 1992; 2000; Puiatti, 2001). Na cv. Lehua Maoli, Shih \& Snyder (1984) verificaram que a área foliar aumentou rapidamente durante os seis primeiros meses, manteve-se relativamente constante por três meses e declinou no último mês, alcançando a produção máxima de rizomas com alto índice de área foliar acumulado.

A determinação da área foliar, utilizando medidas lineares, demonstrou ser possível estimar a mesma por meio de equações de regressão, sem destruir o material, em folhas de taioba (Xanthosoma sagittifolium), (Chapman, 1964) e em taro (Colocasia esculenta), (Nolasco et al., 1983; Goenaga \& Singh, 1996). Resultados demonstram que o índice de área foliar, o qual foi associado com maior produção de matéria seca, é importante atributo fisiológico para estimar o rendimento em Colocasia (Shih \& Snyder, 1984; Roychowdhury, 1995).

Os açúcares resultantes do processo fotossintético são translocados do limbo foliar para os pecíolos, local onde, dependendo do clone, ocorre menor ou maior armazenamento desses açúcares; a translocação prossegue até os rizomas, onde ocorre a síntese do amido de reserva e, consequentemente, o cresci- mento dos rizomas filhos (Hashad et al., 1956). Por essa razão, condições que propiciem às plantas de taro manter as folhas com grandes limbos e pecíolos exuberantes têm o potencial de proporcionarem plantas mais produtivas. Espera-se, portanto, que a quantidade de reservas presentes no rizoma muda tenha influência sobre o crescimento da planta e a produção final de rizomas.

A propagação do taro, na exploração comercial de rizomas, é exclusivamente vegetativa, utilizando-se os rizomas filhos os quais constituem em principal e, na maioria das vezes, único produto para a comercialização (Puiatti, 2001). Apesar do trabalho clássico de Silva (1970), o qual não obteve resposta, em produção de rizomas, com incremento no tamanho de mudas tipo rizomas filhos, outros trabalhos (Bourke \& Perry, 1976; Almeida et al., 1984; Vasconcelos et al., 1986; Katsumoto \& Puiatti, 1990) sugerem que há efeito do tamanho da muda na produção. Todavia, quanto maior o peso médio de rizomas filhos utilizados como muda, maior será o peso desse componente no custo de produção, além do incremento no peso médio das mudas nem sempre levar ao incremento proporcional em produtividade (Puiatti, 2001). Por essa razão, o aproveitamento da cabeça na propagação da cultura, torna-se uma opção interessante, conforme já observado por Almeida et al. (1984) e Vasconcelos et al. (1986).

O objetivo do trabalho foi verificar a viabilidade agronômica e econômica da utilização do rizoma mãe (cabeça) de taro 'Chinês' na implantação da cultura, comparado à propagação convencional (rizomas filhos).

\section{MATERIAL E MÉTODOS}

O trabalho foi conduzido a campo, em área experimental da UFV de 23/09/ 89 a 15/06/1990. Utilizou-se o taro 'Chinês', pertencente ao Banco de Germoplama de Hortaliças da UFV (BGH 5928). O solo utilizado, Podzólico Vermelho-Amarelo Câmbico, textura argilosa e topografia plana, apresentou, nas análises química e física os valores: $\mathrm{pH}$ em água $(1: 2,5)=5,9 ; \mathrm{Al}^{+3}$ $=0,0, \mathrm{Ca}^{+2}=4,2, \mathrm{Mg}^{+2}=1,0, \mathrm{H}+\mathrm{Al}=$
$4,4, \mathrm{SB}=5,75$, CTCef $=5,75 \mathrm{cmol}_{\mathrm{c}} \mathrm{dm}^{-3}$ de solo; $\mathrm{P}=150,6$ e $\mathrm{K}=213,0 \mathrm{mg} \mathrm{dm}^{-3}$ de solo; $\mathrm{m}=0,0$ e $\mathrm{V}=56,7 \%$ e $\mathrm{C}=$ 2,65; argila $=51$, silte $=22$, areia fina $=$ $11 \mathrm{e}$ areia grossa $=16 \mathrm{dag} \mathrm{kg}^{-1}$ de solo.

$\mathrm{O}$ experimento constou de dez tratamentos, dispostos no delineamento blocos ao acaso, com cinco repetições. Os tratamentos consistiram de cabeça central intacta $(\mathrm{CI})$, com peso médio de $300 \mathrm{~g}$; cinco segmentos da cabeça: metade superior (CMS) com $150 \mathrm{~g}$; metade inferior (CMI) com $150 \mathrm{~g}$; terço superior (CTS) com $100 \mathrm{~g}$; terço mediano (CTM) com $100 \mathrm{~g}$; terço inferior (CTI) com $100 \mathrm{~g}$; metade lateral (CML) com $150 \mathrm{~g}$; e três classes de rizoma: rizoma filho grande (RFG) com $100 \mathrm{~g}$; rizoma filho médio (RFM) com $50 \mathrm{~g}$ e rizoma filho pequeno (RFP) com $25 \mathrm{~g}$.

A parcela foi composta de três linhas com 4,2 m de comprimento cada, distanciadas de $1,0 \mathrm{~m}$, contendo 14 plantas espaçadas de $0,3 \mathrm{~m}$, totalizando uma área de $12,6 \mathrm{~m}^{2}$. A área útil foi constituída pela linha central, excetuando-se 0,6 $\mathrm{m}$ nas extremidades, com $3,0 \mathrm{~m}^{2}$.

No solo, após aração e gradagem, foram abertos sulcos de plantio, com sulcador bico de pato, numa profundidade aproximada de $12 \mathrm{~cm}$. As mudas foram colocadas nos sulcos e cobertas com solo, ficando cerca de 6 a $8 \mathrm{~cm}$ de profundidade. Em razão dos níveis elevados de nutrientes apresentados na análise química do solo não foi realizada a adubação da cultura. Também não foi realizado controle químico. Durante o ciclo de cultivo foram realizadas, em todos os tratamentos, quatro capinas com auxílio de enxadas na linha e nas entrelinhas com o cultivador de enxadas tração animal, aos 35, 68, 101 e 145 dias após o plantio (DAP). Quando da ausência de chuvas até 30 dias antes da colheita, foram realizadas irrigações semanais, por aspersão, aplicando-se, em cada irrigação, lâmina de água de cerca de $40 \mathrm{~mm}$, considerada suficiente para atender as necessidades da cultura (Soares, 1991).

Aos 45 DAP e, posteriormente, a cada 30 dias, avaliou-se o estande de plantas na área total da parcela. Nessas mesmas épocas, em cinco plantas da parcela útil, previamente escolhidas e demarcadas, avaliou-se a área foliar e 


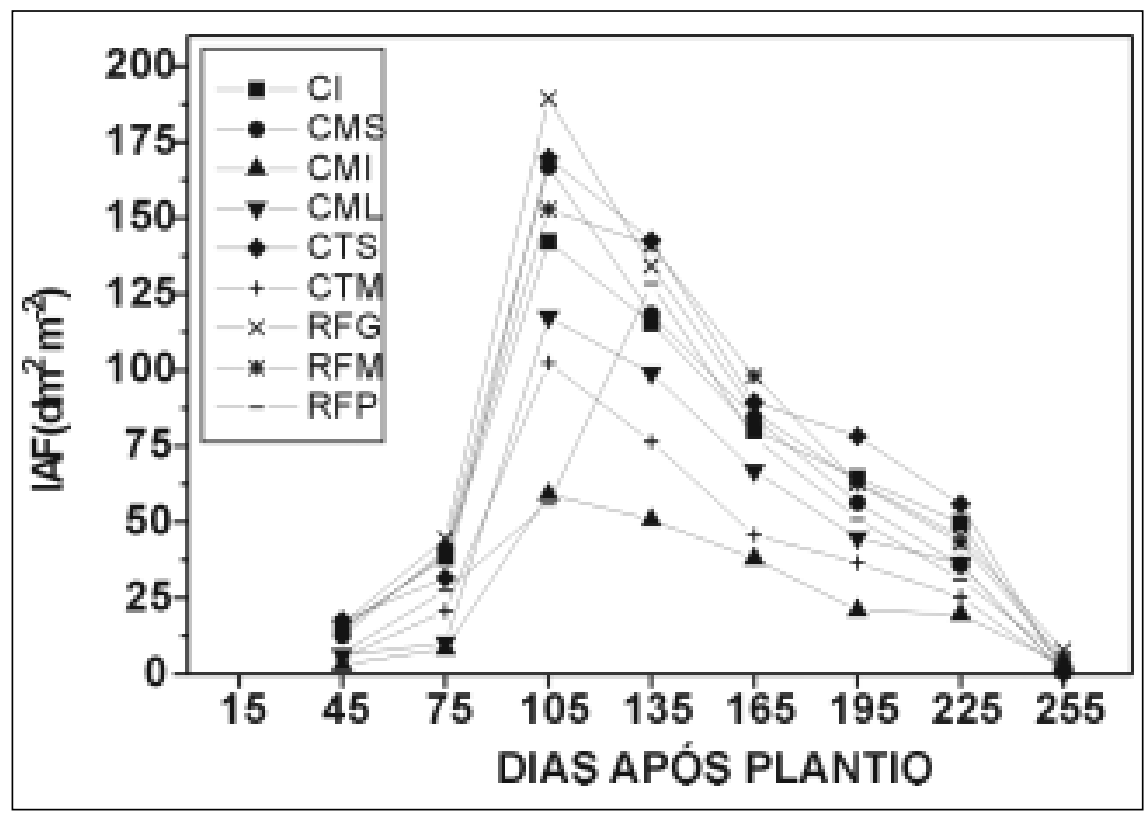

Figura 1. Crescimento, em índice de área foliar (IAF), de plantas de taro 'Chinês' observadas durante o ciclo em função do tipo de muda. Viçosa, UFV, 1989-90. Tipos de mudas: vide legenda da tabela 1 .

altura de planta. A área foliar foi calculada pelo método não destrutivo desenvolvido por Chapmam (1964) e adaptado para o taro 'Chinês' por Nolasco et al. (1983). A altura de planta foi obtida pela distância do nível do solo à inserção do pecíolo no limbo foliar da folha mais alta.

Após nove meses do plantio, atingida a maturação, as plantas foram colhidas e avaliadas quanto à produção de rizomas. Os rizomas mãe (RM) foram separados e os rizomas filhos classificados, com base no diâmetro, de acordo com Puiatti et al. (1990) nas classes filho grande [FG (>47 mm)], filho médio [FM (40-47 mm)], filho pequeno [FP (33$40 \mathrm{~mm})$ ] e refugo [RE $(<33 \mathrm{~mm})]$. Considerou-se o somatório das três primeiras classes como produção comercial.

A análise econômica foi realizada estimando-se os possíveis retornos obtidos com a venda de rizomas, de cada classe, na CEASA-MG entre junho a setembro (comercialização dos rizomas colhidos).

O retorno líquido foi calculado descontando-se, no retorno total estimado obtido, o custo das mudas, além dos demais encargos de produção. No custo das mudas foram considerados, além do próprio custo dessas, os gastos com serviços de mão-de-obra na segmentação das cabeças. Nos tipos de mudas em que se procedeu à segmentação das cabeças, o custo da operação foi rateado entre os tipos de segmentos obtidos e os demais encargos foram considerados idênticos para todos os tratamentos, sendo estimado em R \$1.200,00/ha. Não se considerou, nos cálculos, o custo da terra e os gastos com embalagens e as irrigações esporádicas. Para essas análises, tanto de venda das classes de rizomas quanto das mudas utilizadas, trabalhouse com os valores médios de preço/kg praticado na CEASA-MG nos meses de junho a setembro de 2001, sendo estes de $\mathrm{R} \$ 0,20, \mathrm{R} \$ 0,55, \mathrm{R} \$ 0,45$ e $\mathrm{R} \$ 0,30$, para rizomas mãe (cabeça), filhos grande, médio e pequeno, respectivamente.

Em decorrência da não obtenção de plantas do tratamento do tipo de muda CTI, as análises estatísticas dos dados obtidos foram realizadas considerandose apenas nove tratamentos. Os dados obtidos foram submetidos à análise de variância e as médias foram comparadas com o teste de Scott-Knott a 5\% de probabilidade.

\section{RESULTADOS E DISCUSSÃO}

População e crescimento de plantas

A população de plantas permaneceu inalterada durante o ciclo, chegando-se à colheita com estande de $92 \%$ (CI), 96\% (CMS), 35\% (CMI), 88\% (CML), 94\% (CTS), 68\% (CTM), 0\% (CTI), $78 \%$ (RFG), $88 \%$ (RFM) e $82 \%$ (RFP). Os tipos de mudas envolvendo as porções inferior (CTI e CMI) e mediana (CTM) da cabeça, foram os que proporcionaram as menores populações de plantas. As falhas dos tratamentos CTI e CMI podem ser explicadas pelo número ínfimo de gemas na porção inferior dos rizomas (Puiatti, 2001).

Com relação ao tipo de muda CTM, a grande área de tecido exposta pelos cortes no sentido equatorial, nas porções basal e adaxial, provavelmente favoreceu a podridão, resultando também em baixo estande, porém maior que dos tipos CTI e CMI. A fragmentação da cabeça pode levar à obtenção de segmentos deficitários em gemas vegetativas, resultando em menores percentuais de emergência, como observado por Vasconcellos et al. (1984) com aumento do número de segmentos obtidos por cabeça. Chuvas ocorridas durante a fase inicial pós-plantio podem ter se constituído em fator adicional na perda desses tipos de mudas. Todavia não explicam o estande reduzido observado nas mudas rizomas filhos e, dentre essas, o fato do tipo de muda RFG ter apresentado menor \% de plantas emergidas que o tipo RFM; também não explicam o alto estande proporcionados pelas mudas CMS e CTS, com $96 \%$ e $94 \%$ de plantas emergidas, respectivamente. Essas mudas (CMS e CTS) são segmentos da porção superior da cabeça, tendo grande porção da área basal exposta pelo corte da cabeça no sentido equatorial, fato que, associado ao não tratamento químico, era de se esperar menores porcentagens de plantas emergidas que da muda RFG, uma vez que esses três tipos de mudas possuem gema apical e as mudas CTS e RFG se eqüivalem em termos de reservas.

Independente do tipo de muda as plantas apresentaram crescimento em altura, durante o ciclo de cultivo, bastante semelhantes, destacando-se as plantas oriundas das mudas CI e RFG, como mais altas e CMI, CTM e RFP como as mais baixas (Dados não mostrados). A presença da gema apical nas mudas CI e RFG, associado a maior 
Tabela 1. Rendimento ( $\left.\mathrm{t} \mathrm{ha}^{-1}\right)$ de rizomas mãe (RM), filho grande (FG), filho médio (FM), filho pequeno (FP), comercial (CO), refugo (RE) e total (RT) de taro 'Chinês' em função do tipo de muda. Viçosa, UFV, 1989-90.

\begin{tabular}{|c|c|c|c|c|c|c|c|}
\hline $\begin{array}{c}\text { Tipo de } \\
\text { muda }^{1}\end{array}$ & RM & FG & FM & FP & $\mathrm{CO}$ & RE & RT \\
\hline $\mathrm{Cl}$ & $12,34 \mathrm{a}$ & $3,21 \quad c$ & $14,14 a$ & $8,42 a$ & 25,77 a & $1,01 \mathrm{a}$ & 39,12 a \\
\hline CMS & $8,83 a$ & $3,79 \mathrm{~b}$ & 13,83 a & $7,30 \mathrm{a}$ & 24,93 a & $0,82 a$ & 34,57 a \\
\hline CMI & $5,59 \quad b$ & 1,95 & $5,13 \quad b$ & 3,05 & $10,13 \quad c$ & $0,49 a$ & 16,20 \\
\hline CML & $10,24 a$ & $4,79 a$ & $13,60 \mathrm{a}$ & $6,44 a$ & $24,83 a$ & $0,88 a$ & 35,95 a \\
\hline CTS & $9,11 \mathrm{a}$ & $5,35 a$ & $13,37 \mathrm{a}$ & $6,48 \mathrm{a}$ & $25,20 \mathrm{a}$ & $0,73 \mathrm{a}$ & $35,08 \mathrm{a}$ \\
\hline CTM & $9,22 \mathrm{a}$ & 1,83 & $10,28 \mathrm{a}$ & $5,65 \quad b$ & $17,76 \quad b$ & $0,77 \mathrm{a}$ & $27,74 \quad b$ \\
\hline RFG & $11,24 \mathrm{a}$ & $5,26 a$ & $12,61 \mathrm{a}$ & 5,79 b & 23,66 a & $0,89 a$ & 35,79 a \\
\hline RFM & $10,00 \mathrm{a}$ & $4,21 \quad b$ & $12,88 \mathrm{a}$ & 5,39 b & $22,48 \mathrm{a}$ & $0,81 \mathrm{a}$ & $33,29 a$ \\
\hline RFP & $7,23 \quad b$ & 2,74 & $10,80 \mathrm{a}$ & $5,00 \quad b$ & $18,54 \quad b$ & $0,53 a$ & $26,30 \quad b$ \\
\hline$\overline{C V}(\%)$ & 21,9 & 24,7 & 23,0 & 21,3 & 14,9 & 44,5 & 13,1 \\
\hline
\end{tabular}

*/Médias, nas colunas, seguidas da mesma letra não diferem entre si a 5\% de probabilidade pelo teste de Scott-Knott.

$1 / \mathrm{CI}=$ cabeça intacta $(300 \mathrm{~g}) ; \mathrm{CMS}=$ metade superior da cabeça $(150 \mathrm{~g}) ; \mathrm{CMI}=$ metade inferior da cabeça $(150 \mathrm{~g}) ; \mathrm{CML}=$ metade lateral da cabeça $(150 \mathrm{~g}) ; \mathrm{CTS}=$ terço superior da cabeça $(100 \mathrm{~g}) ; \mathrm{CTM}=$ terço mediano da cabeça $(100 \mathrm{~g}) ; \mathrm{CTI}=$ terço inferior da cabeça $(100 \mathrm{~g})$; $\mathrm{RFG}=$ rizoma filho grande $(100 \mathrm{~g}) ; \mathrm{RFM}=$ rizoma filho médio $(50 \mathrm{~g}) ; \mathrm{RFP}=$ rizoma filho pequeno $(25 \mathrm{~g})$.

quantidade de reservas em relação ao tipo de muda RFP, podem ser as razões do maior crescimento, em altura, proporcionado por essas mudas.

Mudas sem a presença da gema apical (CMI e CTM), foram as que proporcionaram plantas menos vigorosas apresentando, durante o ciclo, menor IAF (Figura 1), enquanto que maior IAF foi observado para plantas oriundas de mudas RFG, CI, CMS, CTS e RFM. Plantas oriundas do tipo de muda RFP, em razão da menor quantidade de reservas, apresentaram crescimento lento na fase inicial, com IAF semelhante às plantas oriundas das mudas CMI e CTM (essas sem a presença da gema apical), todavia se recuperaram após os 105 DAP (Figura 1). Quanto a muda CML, apesar de não apresentar a gema apical devido à natureza polar do corte, segmentando-a longitudinalmente ao meio, por possuir grande quantidade de gemas laterais na sua porção superior, aliado à quantidade de reservas, proporcionou IAF intermediário entre mudas oriundas de segmentos da cabeça com gema apical (CI, CMS e CTS) e sem gema apical (CMI e CTM).

Exceto para RFP que apresentou maior IAF aos 135 DAP, aos 105 DAP foram observados os maiores valores de IAF para os demais tipos de mudas (Figura 1) e aos 165 DAP os maiores valores, em altura de plantas, em praticamente todos os tipos de mudas (Dados não mostrados). Bourk \& Perry (1976) também obtiveram, na fase inicial do ciclo, plantas com maiores valores em altura, número de folhas e de rizomas/planta e de área foliar/planta, oriundas de mudas tipo "huli" com maior diâmetro, evidenciando maior taxa de crescimento em plantas oriundas de mudas com maior quantidade de reservas.

Os resultados de crescimento de parte aérea obtidos estão de acordo com observações de Shih \& Snyder (1984). Puiatti et al. (2000) também observaram, no tratamento controle (monocultivo do taro), maior IAF aos 160 DAP, enquanto que maior altura de planta ocorreu aos 190 DAP. Portanto o IAF máximo é alcançado primeiro, o que faz com que as plantas cresçam em altura à procura de espaço e luz, alcançando altura máxima posteriormente ao IAF máximo.

Pode-se evidenciar, por esses resultados de crescimento, que o rizoma muda exerce efeito direto no crescimento e vigor das plantas e que não se deve balizar apenas na quantidade de reservas do rizoma visando obter plantas vigorosas, pois a presença da gema apical na muda também é um fator de extrema importância no crescimento da planta, sobretudo em termos de área foliar.

Produção de rizomas e retorno estimado

A baixa porcentagem de plantas emergidas e, conseqüentemente, meno- res estandes nos tratamentos CMI, CTM e RFP proporcionaram menores produtividades de rizomas (Tabela 1). Contudo, quando se analisou a produção em peso e número de rizomas por planta, não houve diferença significativa para as classificações efetuadas (Dados não mostrados), evidenciando que em taro parece não haver compensação, em produção de rizomas, pelas plantas remanescentes em condições de menor estande. Falta de compensação, em produção de raízes tuberosas, sob condições de menor população na colheita, também foi observado por Câmara (1994) e Gil Leblanc (2000) em mandioquinhasalsa, demonstrando que esse pode ser um fato comum entre as hortaliças raiz.

A grande variabilidade dos dados relacionados a classes de rizomas, e conseqüentemente, elevados valores de coeficientes de variação, reduziram as chances de detectar diferenças significativas. Na maioria dos casos, mesmo procedendo-se a transformação dos dados para realização das análises a falta de significância persiste. Esse fato é mais evidente nas classes extremas, FG e RE, as quais têm, na sua classificação por diâmetro, delimitados apenas os limites inferior e superior, respectivamente. Bourk \& Perry (1976) também obtiveram, além de menor crescimento de plantas na fase inicial, menor produção de rizomas em plantas oriundas de mu- 
Tabela 2. Custo $\left(\mathrm{R} \$ \mathrm{ha}^{-1}\right)$ de mudas $(\mathrm{CM})$ e retorno estimado $\left(\mathrm{R} \$ \mathrm{ha}^{-1}\right)$ obtido da possível venda da produção de rizomas mãe $(\mathrm{RM})$ e das classes de rizomas filho grande (FG), filho médio (FM), filho pequeno (FP), comercial (CO), total (RT) e retorno líquido (RL) de taro 'Chinês', em função do tipo de muda. Viçosa, UFV, 1989-90.

\begin{tabular}{|c|c|c|c|c|c|c|c|c|}
\hline $\begin{array}{l}\text { Tipo de } \\
\text { muda }^{1}\end{array}$ & CM & RM & FG & FM & FP & $\mathrm{CO}$ & RT & $\mathbf{R L}^{2}$ \\
\hline $\mathrm{Cl}$ & $2.000,00$ & $2.468,49 a$ & $1.764,02 \quad \mathrm{c}$ & $6.361,27 a$ & $2.526,50 \mathrm{a}$ & $10.651,80 \mathrm{a}$ & $13.120,29 \mathrm{a}$ & $9.920,29 a$ \\
\hline CMS & $1.100,00$ & $1.765,69 \mathrm{a}$ & $2.086,75 b$ & $6.223,24 \mathrm{a}$ & $2.190,44 \mathrm{a}$ & $10.500,43$ a & $12.266,12 \mathrm{a}$ & $9.966,12 \mathrm{a}$ \\
\hline CMI & $1.100,00$ & $1.117,56 \quad b$ & $1.070,88$ & $2.307,46 \quad b$ & $916,18 \quad c$ & $4.294,53 \quad c$ & $5.412,08 \quad c$ & $3.112,08$ \\
\hline CML & $1.100,00$ & $2.048,41 a$ & $2.633,19 a$ & $6.118,22 \mathrm{a}$ & $1.932,39 a$ & $10.683,80 \mathrm{a}$ & $12.732,21 \mathrm{a}$ & $10.432,21 \mathrm{a}$ \\
\hline CTS & 733,34 & $1.821,70 \mathrm{a}$ & $2.941,26 a$ & $6.016,20 \mathrm{a}$ & $1.944,39 a$ & $10.901,85 a$ & $12.723,54 \mathrm{a}$ & $10.789,87 a$ \\
\hline СТM & 733,34 & $1.843,04 \mathrm{a}$ & $1.008,54 \quad d$ & $4.623,93 a$ & $1.694,34 \mathrm{~b}$ & $7.326,80 \quad b$ & $9.169,83 \quad b$ & $7.236,16 \quad b$ \\
\hline RFG & $1.832,32$ & $2.247,12 \mathrm{a}$ & $2.893,58 \mathrm{a}$ & $5.674,13 a$ & $1.736,35 \mathrm{~b}$ & $10.304,06$ a & $12.551,18 \mathrm{a}$ & $9.517,86 \mathrm{a}$ \\
\hline RFM & 750,00 & $1.999,07 \mathrm{a}$ & $2.317,80 \quad b$ & $5.794,16$ a & $1.616,32 b$ & $9.728,28 \mathrm{a}$ & $11.727,34 \mathrm{a}$ & $9.777,34$ a \\
\hline RFP & 250,00 & $1.445,62 \mathrm{~b}$ & $1.507,30$ & $4.860,97 \mathrm{a}$ & $1.500,30 \mathrm{~b}$ & $7.868,57 \quad b$ & $9.314,20 \quad b$ & $7.864,20 \quad b$ \\
\hline$\overline{C V}(\%)$ & - & 21,9 & 24,7 & 23,1 & 21,4 & 15,4 & 13,7 & 15,1 \\
\hline
\end{tabular}

*/Médias, nas colunas, seguidas da mesma letra não diferem entre si a 5\% de probabilidade pelo teste de Scott-Knott. CM não foi realizada análise estatística. ${ }^{1 / V i d e ~ l e g e n d a ~ d a ~ t a b e l a ~} 1 .{ }^{2} / \mathrm{RL}=\mathrm{RT}-\mathrm{CM}-1.200,00$.

das "huli" com menor diâmetro, evidenciando que a quantidade de reservas na muda é importante atributo relacionado com a taxa de crescimento inicial da planta e com a produção final de rizomas.

Os tipos de mudas que apresentaram piores rendimentos e retorno estimado foram CMI, CTM e RFP (Tabelas 1 e 2). Os segmentos da cabeça CML e CTS apresentaram como grande vantagem os maiores rendimentos de rizomas da classe FG (Tabela 1) e retorno estimado (Tabela 2), não diferindo do tipo de muda RFG, devido aos elevados valores percentuais de participação da classe FG em peso e valor.

A classe FG apresenta maior valor de comercialização (Puiatti, 2001), portanto, é desejável maior percentual de participação dessa classe na produção total, uma vez que possibilitará maior retorno. Nesse aspecto os tipos de mudas CTS, RFG e CML, nessa ordem, foram os que apresentaram os maiores valores de participação na classe FG, tanto em produção de rizomas quanto em valor de comercialização (Tabelas 1 e 2). Dentre esses, o tipo de muda CTS mostrou ser mais eficiente em termos de produção de rizomas da classe FG que os tipos de muda CML e RFG, pois acumulou menos reserva na cabeça (Tabela 1), ou seja, a razão matéria fresca produzida de FG/RM foi de 0,59 no tipo de muda CTS, enquanto que nos tipos CML e RFG essa razão foi de 0,47.
Pode-se observar que, além do aspecto de população, o crescimento e vigor das plantas estão relacionados com o tipo de muda. Tipos de mudas que proporcionaram maiores índices de área foliar (Figura 1), também proporcionaram maiores produções de rizomas e RL (Tabelas 1 e 2), conforme observações de Reddy et al. (1968), Shih \& Snyder (1984), Roychowdhury (1995). Segundo Hashad et al. (1956) os açúcares resultantes do processo fotossintético são translocados do limbo foliar para os pecíolos, e desses prossegue até os rizomas, onde ocorre a síntese do amido de reserva e, conseqüentemente, o crescimento dos rizomas filhos. Por essa razão, tipos de mudas que propiciaram plantas com maior IAF e mais altas foram mais produtivas.

Pelos resultados obtidos, pode-se concluir que a utilização da cabeça intacta (CI) ou dos segmentos CMS, CML e CTS na propagação do taro 'Chinês' é viável em termos agronômicos, corroborando com as observações de Almeida et al. (1984).

Em termos econômicos, computandose o custo das mudas, os tipos CI, CMS, CML, CTS, RFM e RFG, demonstraram retorno líquido (RL) maior que os tipos de muda CTM, CMI e RFP. Se computar os custos operacionais de preparo (limpeza), embalagem e transporte da classe RM, provavelmente o retorno se diferirá, devido ao baixo valor de comercialização e de aceitação da cabeça, evidenciando a van- tagem de se comercializar as classes de rizomas filhos e de se utilizar a cabeça na propagação. No caso da não existência de mercado para a classe RM, a utilização da cabeça na propagação passa ser extremamente vantajosa, pois o custo dessas mudas praticamente deixa de existir, proporcionando maior retorno líquido. Nessa última situação, o agricultor que possui na propriedade criação de suínos poderá fazer a utilização dos segmentos CMS ou CTS na propagação da cultura podendo obter altas produtividade e retorno econômico além do aproveitamento dos segmentos inferiores (metade e $2 / 3$ inferiores da cabeça) na alimentação desses animais.

\section{LITERATURA CITADA}

ALMEIDA, D.L.; VASCONCELLOS, H.O.; PESSANHA, G.G. Épocas de plantio e tipos de mudas na cultura do inhame (Colocasia esculenta Schott). In: CONGRESSO BRASILEIRO DE OLERICULTURA, XXIV, 1984, Jaboticabal, SP. Resumos... Jaboticabal: FCAV, 1984. p. 163.

BOURK, R.M.; PERRY, C.H. Influence of set size on growth and yield of taro (Colocasia esculenta). Papua New Guinea Agricultural Journal, v. 27, n. 4, 1976.

CÂMARA, F.L.A. Efeito da competição entre plantas na produção de mandioquinha-salsa. Horticultura Brasileira, Brasília, v. 12, n. 1, p. 74, 1994. Resumo.

CAMARGO FILHO; MAZZEI, A.R.; ALVES, H.S. Mercado de raízes e tubérculos: análise de preços. Informações Econômicas, São Paulo, v. 13, n. 2, 2001.

CHAPMAN, T. A Note on the measurement of leaf area of the tannia (Xanthosoma sagittifolium). Tropical Agriculture, v. 41, n. 4, p. 351-352, 1964. 
FAO. FAO statistical database. Disponível em $<$ http//www.fao.org/>. Acesso em 29/11/2001. GIL LEBLANC, R.E. Crescimento e produção de clones de batata-baroa (Arracacia xanthorrhiza Bancroft), influenciados por pré-enraizamento $e$ tipo de muda. Viçosa: UFV, 2000. 100 p. (Tese mestrado).

GOENAGA, R.; SINGH, U. Estimation of leaf area of taro [Colocasia esculenta (L.) Schott] from linear measurements. The Journal of Agriculture of the University of Puerto Rico, v. 80, n. 3, p. 183-185, 1996.

HEREDIA ZÁRATE, N.A. Curvas de crescimento de inhame (Colocasia esculenta (L.) Schott), considerando cinco populações em solo seco e alagado.Viçosa: UFV, 1988. 95 p. (Tese doutorado).

HASHAD, M.N.; STINO, K.R.; EL-HINNAMY, S.I. Transformation and translocation of carboidrates in taro plants during growth. Annals of Agricultural Science, v. 1, n. 1, 1956.

KATSUMOTO, R.; PUIATTI, M. Utilização da cabeça central como rizoma-muda na cultura do inhame (Colocasia esculenta) 'Chinês'. In: RESUMOS DO CONGRESSO BRASILEIRO DE OLERICULTURA, XXX. Campo Grande, MS. 1990. Horticultura Brasileira, Brasília, v. 8. n. 1, p. 47, 1990. Resumo 094.

NIP, WAI-KIT. Taro food products. In: HOLLYER, J.R. \& SATO, D.M., ed. Taking taro into the 1990: a taro conference. Proceedings... Hawaii: University of Hawaii, 1990. p. 3-5.
NOLASCO, F.; CASALI, V.W.D.; CONDÉ, A.R. Avaliação da área foliar do inhame (Colocasia esculenta Schott.) no campo. In: CONGRESSO BRASILEIRO DE OLERICULTURA, 23, Rio de Janeiro-RJ, 1983. Resumos S.O.B. p. 19.

PLUCKNETT, D.L. Taxonomy of the Genus Colocasia. In: WANG, J.K., HIGA, S. ed. Taro: A review of Colocasia esculenta and its potentials. Honolulu: University of Hawaii Press, 1983. p. 14-19.

PUIATTI, M. Manejo da cultura do taro. In: CARMO, C.A.S. (Ed.). Inhame e taro: sistemas de produção familiar. Vitória-ES: INCAPER, 2001. p. 203-252.

PUIATTI, M.; CAMPOS, J.P.; CASALI, V.W.D.; CARDOSO, A.A.; CRUZ, R. Sistema de colocação do bagaço de cana-de-açúcar e do capim gordura, na cultivar de inhame Chinês. Horticultura Brasileira, Brasília, v. 8, n. 1, p. 14-16, 1990. PUIATTI, M.; FÁVERO, C.; FINGER, F.L.; GOMES, J.M. Crescimento e produtividade de inhame e milho doce em cultivo associado. Horticultura Brasileira, Brasília, v. 8, n. 1, p. 2430, 2000.

PUIATTI, M.; GREEMAN, S.; KATSUMOTO, R.; FÁVERO, C. Crescimento e absorção de macronutrientes pelo inhame 'Chinês' e 'Japonês'. Horticultura Brasileira, Brasília, v. 10, n. 2, p. 89-92, 1992.

REDDY, V.B., MEREDITH, W.F., BROWN, B.T.

A Note on the relationship between corm yield and certain leaf measurements in taro [Colocasia esculenta (L.) Schoot]. Tropical Agriculture, v. 45, n. 3, p. 243-245, 1968.
ROYCHOWDHURY, S. Leaf area development in colocasia and its relationship with yield. Indian Journal Plant Physiology, v. 38, n. 4, p. 305-308, 1995.

SILVA, J.F. Espaçamento, adubação e tamanho de mudas na produção de inhame (Colocasia esculenta Schott). Viçosa: UFV, 1970. 40 p. (Tese mestrado).

SHIH, S.F., SNYDER, G.H. Leaf area index and dry biomass of taro. Agronomy Journal, v. 76, p. 750-753, 1984.

SOARES, J.G. Crescimento do inhame (Colocasia esculanta (L.) Schott) em duas condições agroclimáticas, em seis níveis de água e cobertura morta. Viçosa: UFV, 1991. 91p. (Tese mestrado).

STANDAL, B.R. Nutritive value. In: WANG, J.K., HIGA, S. ed. Taro: A review of Colocasia esculenta and its potentials. Honolulu: University of Hawaii Press, 1983. p. 141-147.

VASCONCELLOS, H.O.; SOUZA, J.P.; COELHO, R.G.; LEAL, N.R. Propagação de inhame (Colocasia esculenta) através de diferentes tipos de fragmentos da cabeça central. In.: RESUMOS DO CONGRESSO BRASILEIRO DE OLERICULTURA, XXVI, 1986, Salvador, BA. Horticultura Brasileira, Brasília, v. 4, n. 1, p 75, 1986. Resumo 185.

WANG, J.K. Introduction. In: WANG, J.K., HIGA, S. ed. Taro: A review of Colocasia esculenta and its potentials. Honolulu: University of Hawaii Press, 1983. p. 3-13. 